\title{
The temporal effect of platelet-rich plasma on pain and physical function in the treatment of knee osteoarthritis: systematic review and meta-analysis of randomized controlled trials
}

Longxiang Shen ${ }^{1 \dagger}$, Ting Yuan ${ }^{1 \dagger}$, Shengbao Chen ${ }^{2}, X_{\text {Xuetao } X i e^{1 *} \text { and Changqing Zhang }}{ }^{1}$

\begin{abstract}
Background: Quite a few randomized controlled trials (RCTs) investigating the efficacy of platelet-rich plasma (PRP) for treatment of knee osteoarthritis (OA) have been recently published. Therefore, an updated systematic review was performed to evaluate the temporal effect of PRP on knee pain and physical function.

Methods: Pubmed, Embase, Cochrane library, and Scopus were searched for human RCTs comparing the efficacy and/or safety of PRP infiltration with other intra-articular injections. A descriptive summary and quality assessment were performed for all the studies finally included for analysis. For studies reporting outcomes concerning Western Ontario and McMaster Universities Arthritis Index (WOMAC) or adverse events, a random-effects model was used for data synthesis.

Results: Fourteen RCTs comprising 1423 participants were included. The control included saline placebo, HA, ozone, and corticosteroids. The follow-up ranged from 12 weeks to 12 months. Risk of bias assessment showed that 4 studies were considered as moderate risk of bias and 10 as high risk of bias. Compared with control, PRP injections significantly reduced WOMAC pain subscores at 3,6 , and 12 months follow-up $(p=0.02,0.004,<0.001$, respectively); PRP significantly improved WOMAC physical function subscores at 3,6 , and 12 months $(p=0.002,0.01,<0.001$, respectively); PRP also significantly improved total WOMAC scores at 3,6 and 12 months (all $p<0.001$ ); nonetheless, PRP did not significantly increased the risk of post-injection adverse events $(\mathrm{RR}, 1.40[95 \% \mathrm{Cl}$, 0.80 to 2.45$], P^{2}=59 \%, p=0.24$ ).

Conclusions: Intra-articular PRP injections probably are more efficacious in the treatment of knee OA in terms of pain relief and self-reported function improvement at 3,6 and 12 months follow-up, compared with other injections, including saline placebo, HA, ozone, and corticosteroids.
\end{abstract}

Review registration: PROSPERO CRD42016045410. Registered 8 August 2016.

Keywords: Platelet-rich plasma, Hyaluronic acid, Knee, Osteoarthritis, Systematic review

\footnotetext{
*Correspondence: xuetaoxie@163.com

${ }^{\dagger}$ Equal contributors

'Department of Orthopaedic Surgery, Shanghai Sixth People's Hospital

affiliated to Shanghai Jiaotong University School of Medicine, 600 Yishan

Road, Shanghai 200233, China

Full list of author information is available at the end of the article
} 


\section{Background}

Osteoarthritis (OA) is a major cause of knee disability involving cartilage damage related to an inadequate healing response in the inflammatory milieu [1]. Current non-surgical treatment modalities include physiotherapy, analgesia, non-steroidal anti-inflammatory drugs, and intra-articular injections, such as hyaluronic acid (HA), corticosteroids, or Ozone, with the purpose of reducing symptoms and improving joint function [2-4].

In the past decade, there has been an increasing interest in the use of autologous growth factors, such as intra-articular injections of platelet-rich plasma (PRP) for treatment of knee OA [5]. PRP is a fraction of whole blood and prepared by the centrifugation of autologous blood, thereby yielding a higher concentration of platelets than baseline values. The regenerative effect and anti-inflammatory potential of PRP in the tissue healing process have led to extensive investigation of PRP as a potential treatment for a variety of musculoskeletal indications, including OA [6-8].

A number of randomized controlled trials (RCTs) were reported with favourable outcomes of PRP injections [9-17]; several reviews, including systematic reviews and meta-analysis, have been published with conclusion that PRP was found to be an effective and safe orthobiologic in the treatment of knee OA compared with other intra-articular injections [18-28]. However, these reviewers also concluded that more RCTs, in particular high-quality studies, were still needed. Considering that prior reviews either included non-RCTs or only synthesized a small number of RCTs (less than 9) for analysis [18-28] and that quite a few more RCTs recently have been published [29-35], we believe that it is necessary to perform an updated systematic review and meta-analysis, if appropriate, to evaluate whether the evidence-based support for PRP treatment will be strengthened or compromised. Furthermore, a large number of studies may allow us to fully investigate the temporal effect of PRP specifically on knee pain and physical function.

\section{Methods}

This systematic review was registered online in PROSPERO (registration number: CRD42016045410) and was performed following the guidelines of the PRISMA statement. The protocol and the PRISMA checklist were provided as Additional files 1 and 2, respectively.

\section{Inclusion and exclusion criteria}

All published RCTs evaluating the efficacy and/or safety of PRP (or preparations including autologous platelet concentrate, autologous conditioned plasma, and plasma rich in growth factors) in the treatment of knee OA in human were eligible for inclusion. Only studies that included patients aged 18 years or older with symptomatic knee OA and had a minimum follow-up of 12 weeks were included. All studies had to include at least 1 control group treated by intra-articular agents other than PRP. The studies that PRP was used in combination with operations were excluded. Published abstracts of RCTs without complete data for analysis were also excluded.

\section{Primary and secondary outcomes}

For data synthesis across studies, the primary outcome was the Western Ontario and McMaster Universities Arthritis Index (WOMAC) [36]. Specifically, the WOMAC pain subscores, physical function subscores, and total scores at 3,6 , and 12 months after treatment were recorded. The secondary outcome was the number of patients reporting adverse events.

\section{Search strategy}

Two investigators performed a systematic search of Pubmed, Embase, Cochrane library, and Scopus independently on July 15, 2016 and updated on November 15, 2016. The search strategy was as follows: (platelet[text word] OR plasma[text word]) AND (knee[text word] OR tibiofemoral[text word] OR patellofemoral[text word]) AND (*arthritis[text word] OR *arthritic[text word] OR cartilage[text word] OR *arthrosis[text word] OR gonarthrosis[text word]) AND random*[text word]. In Scopus, the search field [text word] was replaced with [TITLEABS-KEY]. No language or date exclusions were applied (Additional file 3).

Two investigators reviewed all titles and abstracts to remove duplicates and evaluate the relevance according to the inclusion and exclusion criteria. If ambiguity was encountered, the full-text review was performed. Any discrepancy was resolved through panel discussion with a third investigator. The references of prior systematic reviews were also reviewed to find potential eligible studies.

\section{Data extraction}

Two reviewers independently performed data extraction using a pre-developed data extraction table. We extracted the basal characteristics of the included studies to form descriptive summaries. In multi-arm trials including more than one PRP treatment groups, only the group treated with at least twice PRP injections was considered as the intervention group, as the regimen of multiple PRP injections was more common and reported to be more efficacious than a single injection [37, 38]. Although data concerning the patients treated with single-PRP injection in those trials were also extracted, they were not used for quantitative synthesis. The extracted data were checked for consistency, and discrepancies were discussed until a consensus was reached. 
Personal correspondence was attempted to obtain missing data or clarify ambiguous information.

\section{Quality assessment}

Two investigators independently assessed the methodological quality of each eligible study using Review Manager 5.3 (The Cochrane Collaboration, Oxford, England) to determine the risk of bias. The following domains were assessed: random sequence generation (selection bias), allocation concealment (selection bias), blinding of participants (performance bias), blinding of personnel (performance bias), blinding of outcome assessment (detection bias), incomplete outcome data (attrition bias), selective reporting (reporting bias), and other bias. The risk of bias for each domain was graded as either low $(+)$, high (-), or unclear (?) [39]. A trial was regarded as low risk of bias only when all domains were scored as low risk of bias; if 1 or 2 domains were scored as high or moderate risk of bias, the trial was regarded as moderate risk of bias; if more than 2 domains were scored as high or moderate risk of bias, then high risk of bias was considered [21]. Differences were settled by panel discussion with a third investigator.

\section{Data analysis}

For the continuous variables, the mean difference (MD) with $95 \%$ confidence interval (CI) was used, while the relative risk (RR) with 95\% CI was adopted for dichotomous variables to express intervention effects. We assumed the presence of heterogeneity a priori and used the random-effects model in all pooled analysis. The $I^{2}$ was used to test heterogeneity. As defined previously, a value less than $40 \%$ means the heterogeneity might not be important, whereas the value more than $75 \%$ means considerable heterogeneity [39]. To detect the effect of individual studies on the pooled effect, sensitivity analysis was conducted. Publication bias was assessed with a funnel plot if there were at least 10 studies in a comparison [39]. Any $p$ value less than 0.05 was considered to be statistically significant. All analysis was undertaken using Review Manager 5.3.

\section{Results}

Study characteristics

In total, 14 RCTs [9-11, 13-15, 17, 29-35] were included in the analysis published between 2011 and 2016. Details of the literature search were shown in a flowchart (Fig. 1). Search strategy and study selection process could be found in the Additional file 3 .

A total of 1423 patients were included for randomization (Table 1). The sample size of PRP group ranged from 12 to 96 patients, whereas that of control groups including HA, placebo, ozone, and corticosteroids, ranged from 11 to 96 participants. WOMAC was the most commonly used

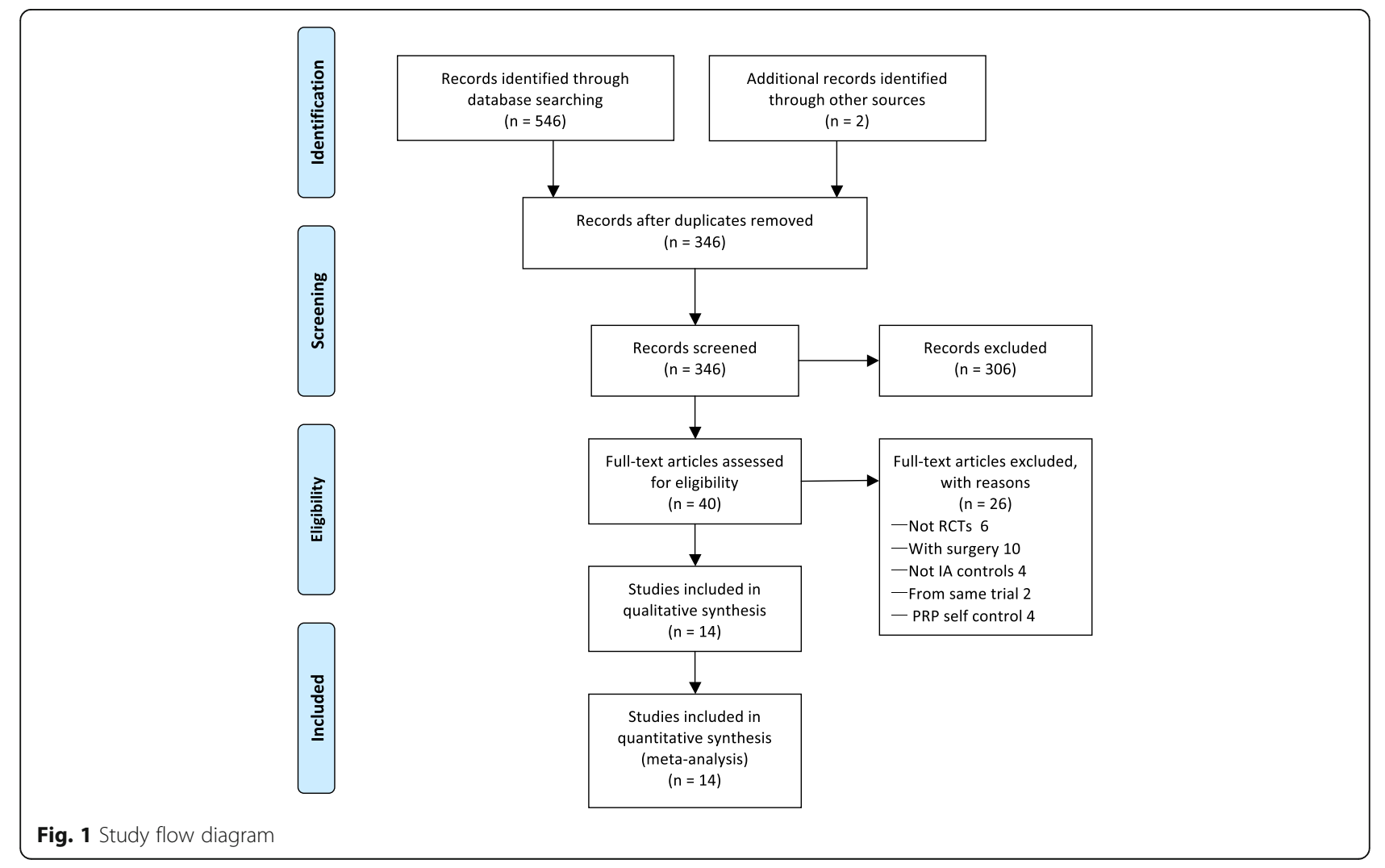




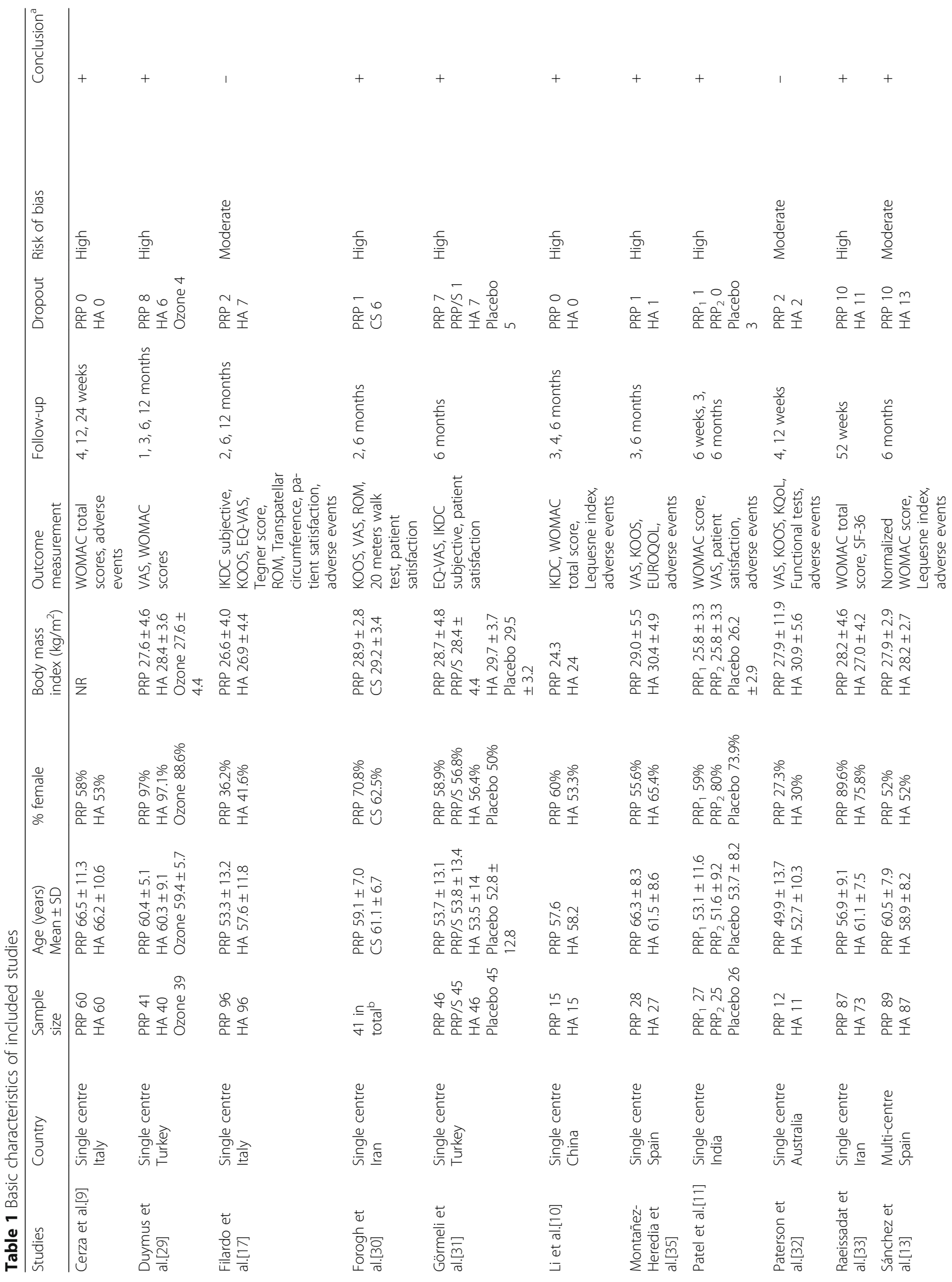




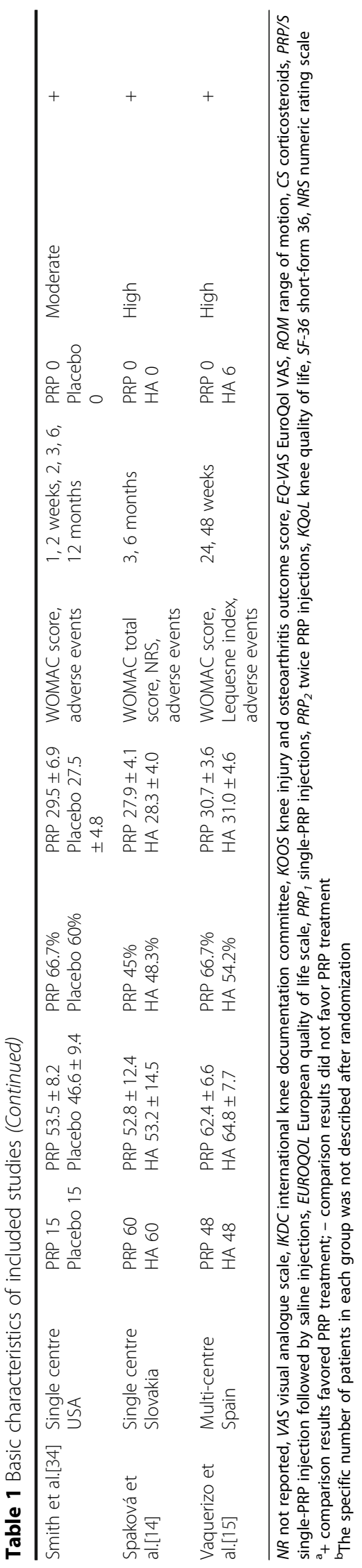


efficacy outcome, and 9 studies reported WOMAC (8 studies) [9-11, 14, 15, 29, 34, 35] or normalized WOMAC (1 study) [13] scores. Follow-up intervals and length were variable among studies. The shortest follow-up was 12 weeks [32] and the longest was 12 months [15, 17, 29, 33, 34]. A summary of PRP intervention effect per study demonstrated comparable efficacy between PRP and HA among 215 patients in 2 studies [17, 32] and superior results in PRP-treated patients compared with control among 1208 patients in the rest 12 studies [9-11, 13-15, 29-31, 33-35].

PRP treatment protocols varied among studies in terms of preparation devices, centrifugations, the use of exogenous activators, and the injection regimen of dose, times, and intervals (Table 2).

Among the 14 studies, 2 different radiographic OA grading systems were used: the Kellgren Lawrence grading (0-IV) [40] in 12 studies $[9,10,14,15,17$, 29-35] and the Ahlbäck scale (I-V) [41] in 2 studies $[11,13]$ (Table 3). According to the distribution of these cases, most participants receiving PRP treatment were at the early or mid-stage of knee OA.

\section{Risk of bias assessment}

A summary of risk of bias assessment of all included studies was illustrated in Fig. 2. Four studies [13, 17, 32, 34] achieved a moderate risk of bias, while the rest 10 [9-11, 14, 15, 29-31, 33, 35] obtained a high risk of bias (Table 1). A detailed justification of the evaluation of each domain of bias was described and provided in the Additional file 4.

\section{Knee pain}

At 3 months, 3 studies reported WOMAC pain subscores, and a statistically significant difference was found in favor of PRP treatment compared with control (MD, -3.69 [95\% CI, -6.87 to -0.51$], I^{2}=94 \%$, $p=0.02$ ). At 6 months, the synthesis of 5 studies demonstrated a statistically significant difference in favor of PRP treatment (MD, -3.82 [95\% CI, -6.40 to -1.25$\left.], I^{2}=96 \%, p=0.004\right)$. At 12 months, the pooling results of 4 studies still favored PRP treatment (MD, -3.76 [95\% CI, -5.36 to -2.16 ] $I^{2}=86 \%$, $p<0.001$ ) (Fig. 3).

Table 2 Details of PRP treatment protocols and control

\begin{tabular}{|c|c|c|c|c|c|c|c|c|}
\hline \multirow[b]{2}{*}{ Studies } & \multicolumn{6}{|l|}{ PRP } & \multicolumn{2}{|l|}{ Control } \\
\hline & Category $^{a}$ & Preparation & Spinning & Activation & $\begin{array}{l}\text { Injection dose, times, } \\
\text { and intervals }\end{array}$ & Fresh/ frozen & Type & $\begin{array}{l}\text { Injection dose, times, } \\
\text { and intervals }\end{array}$ \\
\hline Cerza et al.[9] & LP-PRP & $\mathrm{ACP}$ & Single & NR & $5.5 \mathrm{~mL}, 4$ times, weekly & Fresh & Hyalgan, & 20 mg, 4 times, weekly \\
\hline Duymus et al.[29] & LR-PRP & Ycellbio kit & Single & No & $5 \mathrm{~mL}, 2$ times, monthly & Fresh & $\begin{array}{l}\text { Ostensil Plus, } \\
\text { Ozone gas }\end{array}$ & $\begin{array}{l}40 \text { mg, } 1 \text { time; } \\
15 \text { mL, } 4 \text { times, weekly }\end{array}$ \\
\hline Filardo et al.[17] & LR-PRP & Custom & Double & $\mathrm{CaCl}_{2}$ & $5 \mathrm{~mL}, 3$ times, weekly & Frozen & Hyalubrix, & 30 mg, 3 times, weekly \\
\hline Forogh et al.[30] & LR-PRP ${ }^{b}$ & TUBEX kit & Double & $\mathrm{CaCl}_{2}$ & $5 \mathrm{~mL}, 1$ time & Fresh & Depo Medrol & $40 \mathrm{mg}, 1$ time \\
\hline Görmeli et al.[31] ${ }^{c}$ & LR-PRP & Custom & Double & $\mathrm{CaCl}_{2}$ & $5 \mathrm{~mL}, 3$ times, weekly & $\begin{array}{l}\text { 1Fresh/ } \\
\text { 2Frozen }\end{array}$ & $\begin{array}{l}\text { Orthovisc, } \\
\text { Saline }\end{array}$ & $\begin{array}{l}30 \mathrm{mg}, 3 \text { times, weekly; } \\
\text { NR, } 3 \text { times, weekly }\end{array}$ \\
\hline Li et al.[10] & LR-PRP & Weigao kit & Double & $\mathrm{CaCl}_{2}$ & 3.5 mL, 3 times, 3 weeks & Fresh & Sofast & $2 \mathrm{~mL}, 3$ times, 3 weeks \\
\hline $\begin{array}{l}\text { Montañez-Heredia } \\
\text { et al.[35] }\end{array}$ & LP-PRP & Custom & Double & NR & NR, 3 times, 15 days & Frozen & Adant & NR, 3 times, 15 days \\
\hline Patel et al.[1 1 1 $]^{c}$ & LP-PRP & Custom & Single & $\mathrm{CaCl}_{2}$ & $8 \mathrm{~mL}, 2$ times, 3 weeks & Fresh & Saline & $8 \mathrm{~mL}, 1$ time \\
\hline Paterson et al.[32] & LR-PRP & Custom & Double & Ultraviolet & 3 mL, 3 times, weekly & Fresh & Hylan G-F 20 & $3 \mathrm{~mL}, 3$ times, weekly \\
\hline $\begin{array}{l}\text { Raeissadat } \\
\text { et al.[33] }\end{array}$ & LR-PRP & Rooyagen kit & Double & No & 4-6 mL, 2 times, 4 weeks & Fresh & Hyalgan & 20 mg, 3 times, weekly \\
\hline Sánchez et al.[13] & LP-PRP & PRGF-Endoret & Single & $\mathrm{CaCl}_{2}$ & 8 mL, 3 times, weekly & Fresh & Euflexxa & NR, 3 times, weekly \\
\hline Smith et al.[34] & LP-PRP & ACP & Single & $N R$ & 3-8 mL, 3 times, weekly & Fresh & Saline & 3-8 mL, 3 times, weekly \\
\hline Spaková et al.[14] & LR-PRP & Custom & Triple & No & $3 \mathrm{~mL}, 3$ times, weekly & Fresh & Erectus & NR, 3 times, weekly \\
\hline $\begin{array}{l}\text { Vaquerizo } \\
\text { et al.[15] }\end{array}$ & LP-PRP & PRGF-Endoret & Single & $\mathrm{CaCl}_{2}$ & $8 \mathrm{~mL}, 3$ times, weekly & Fresh & Durolane & NR, 1 time \\
\hline
\end{tabular}

$A C P$ autologous conditioned plasma, $N R$ not reported, $\mathrm{CaCl}_{2}$ calcium chloride, Depo Medrol methylprednisolone acetate injectable suspension, $P R G F$ plasma rich in growth factors

${ }^{a} P R P$ was categorized into two types: LP-PRP (leukocyte-poor PRP) with the level of leukocytes below baseline and LR-PRP (leukocyte-rich PRP) with the level of leukocytes above baseline [45]

${ }^{b}$ Information was obtained from the authors through personal correspondence

In a multi-arm trial, the group injected PRP more than once was regarded as an intervention group, and the data about the single-PRP injection group was not extracted 
Table 3 Radiographic OA grading

\begin{tabular}{|c|c|c|c|c|c|c|c|c|}
\hline \multirow[t]{2}{*}{ Studies } & \multirow[t]{2}{*}{ Intervention } & \multicolumn{4}{|c|}{$\begin{array}{l}\text { Kellgren } \\
\text { Lawrence }\end{array}$} & \multicolumn{3}{|c|}{ Ahlbäck } \\
\hline & & 01 & $\|$ & III & IV & I & $\|$ & III \\
\hline \multirow[t]{2}{*}{ Cerza et al.[9] } & PRP & 21 & 24 & 15 & & & & \\
\hline & $\mathrm{HA}$ & 25 & 22 & 13 & & & & \\
\hline \multirow[t]{3}{*}{ Duymus et al.[29] } & PRP & & 22 & 11 & & & & \\
\hline & $\mathrm{HA}$ & & 24 & 10 & & & & \\
\hline & Ozone & & 23 & 12 & & & & \\
\hline \multirow[t]{2}{*}{ Filardo et al.[17] } & PRP & $\begin{array}{l}0-I V, 1 \\
2.0 \pm 1\end{array}$ & $\begin{array}{l}\text { Mean } \\
1.1\end{array}$ & $\pm S C$ & & & & \\
\hline & $\mathrm{HA}$ & $\begin{array}{l}0-I V, 1 \\
2.0 \pm 1\end{array}$ & $\begin{array}{l}\text { Mean } \\
1.1\end{array}$ & $\pm S C$ & & & & \\
\hline \multirow[t]{2}{*}{ Forogh et al.[30] ${ }^{a}$} & PRP & & 7 & 17 & & & & \\
\hline & CS & & 8 & 16 & & & & \\
\hline \multirow[t]{3}{*}{ Görmeli et al.[31] } & PRP & & l, 26 & & 13 & & & \\
\hline & $\begin{array}{l}\mathrm{PRP} / \mathrm{S} \\
\mathrm{HA}\end{array}$ & & $\begin{array}{l}1,30 \\
1,25\end{array}$ & & $\begin{array}{l}14 \\
14\end{array}$ & & & \\
\hline & Placebo & & l, 27 & & 13 & & & \\
\hline \multirow[t]{2}{*}{ Li et al.[10] } & PRP & 6 & 2 & 4 & 3 & & & \\
\hline & $\mathrm{HA}$ & 6 & 3 & 3 & 3 & & & \\
\hline \multirow{2}{*}{$\begin{array}{l}\text { Montañez-Heredia et } \\
\text { al.[35] }\end{array}$} & PRP & 5 & 10 & 12 & & & & \\
\hline & $\mathrm{HA}$ & 2 & 9 & 15 & & & & \\
\hline \multirow[t]{2}{*}{ Patel et al.[11 ${ }^{a}$} & $\begin{array}{l}\mathrm{PRP}_{1} \\
\mathrm{PRP}_{2}\end{array}$ & & & & & $\begin{array}{l}37 \\
36\end{array}$ & $\begin{array}{l}11 \\
10\end{array}$ & $\begin{array}{l}2 \\
2\end{array}$ \\
\hline & Placebo & & & & & 25 & 18 & 3 \\
\hline \multirow[t]{2}{*}{ Paterson et al.[32] } & PRP & & $\begin{array}{l}\|-\| \\
12\end{array}$ & & & & & \\
\hline & $\mathrm{HA}$ & & $\begin{array}{l}\|-\| \\
11\end{array}$ & & & & & \\
\hline \multirow[t]{2}{*}{ Raeissadat et al.[33] } & PRP & 5 & 34 & 29 & 9 & & & \\
\hline & $\mathrm{HA}$ & 0 & 29 & 23 & 10 & & & \\
\hline \multirow[t]{2}{*}{ Sánchez et al.[13] } & PRP & & & & & 45 & 32 & 12 \\
\hline & $\mathrm{HA}$ & & & & & 42 & 32 & 11 \\
\hline \multirow[t]{2}{*}{ Smith et al.[34] } & PRP & & 8 & 7 & & & & \\
\hline & Placebo & & 10 & 5 & & & & \\
\hline \multirow[t]{2}{*}{ Spaková et al.[14] } & PRP & 2 & 39 & 19 & & & & \\
\hline & $\mathrm{HA}$ & 2 & 37 & 21 & & & & \\
\hline \multirow[t]{2}{*}{ Vaquerizo et al.[15] } & PRP & & 14 & 26 & 8 & & & \\
\hline & $\mathrm{HA}$ & & 18 & 21 & 9 & & & \\
\hline
\end{tabular}

$S D$ standard deviation, $P R P / S$ single-PRP injection followed by saline injections, PRP1 single-PRP injection, PRP2 twice PRP injections

${ }^{\mathrm{a}}$ The number of knees rather than patients was reported

\section{Physical function}

At 3 months, 3 studies reported WOMAC physical function subscores, and a statistically significant difference was found in favor of PRP treatment compared with control (MD, -14.24 [95\% CI, -23.43 to -5.05 ], $I^{2}=91 \%$, $p=0.002)$. PRP treatment was also found to improve physical function significantly according to the pooling

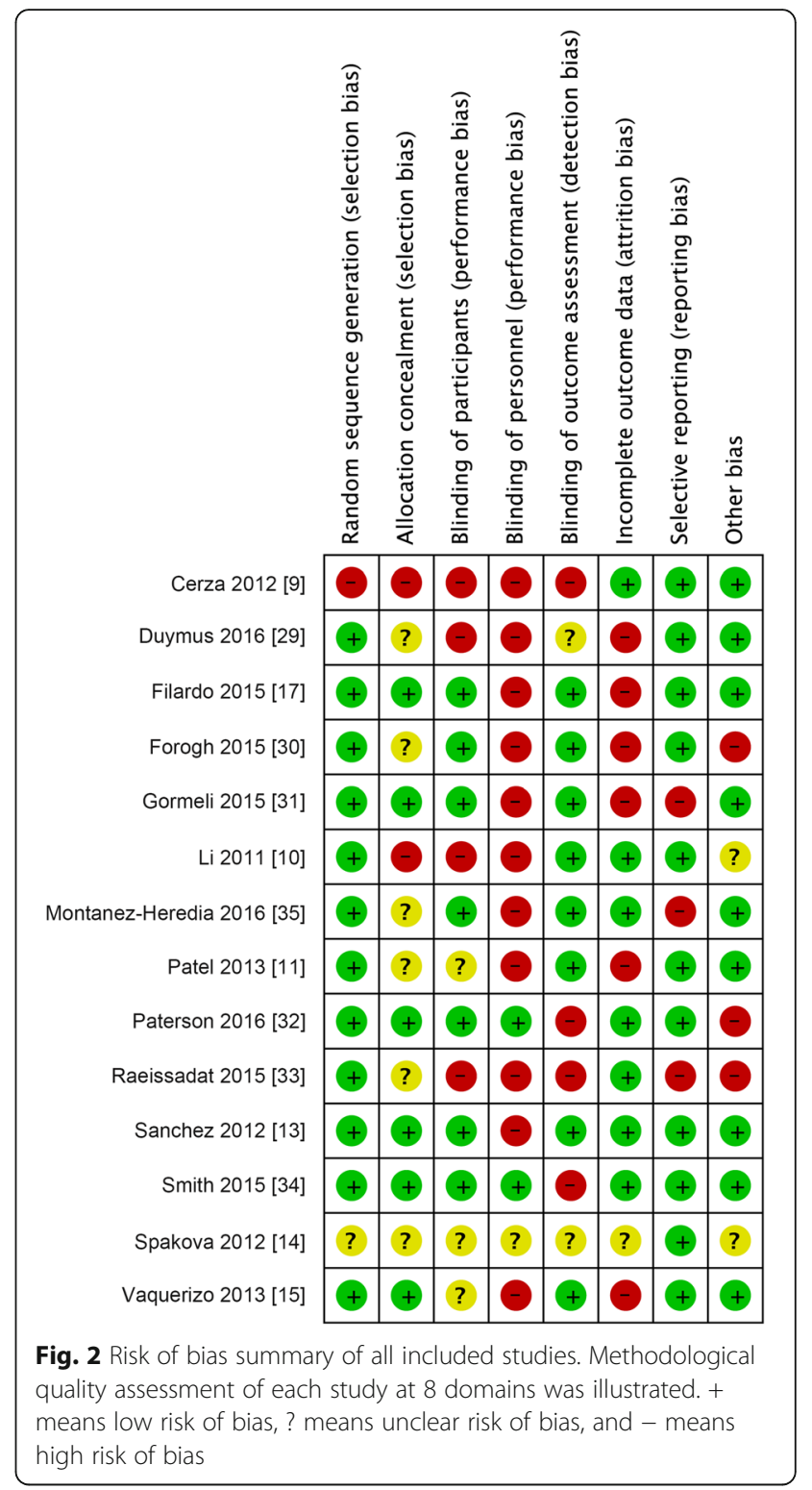

analysis of 5 studies at 6 months (MD, -13.51 [95\% CI, -23.77 to -3.26$\left.], I^{2}=97 \%, p=0.01\right)$ and 4 studies at 12 months (MD, -13.96 [95\% CI, -18.64 to -9.28 ], $I^{2}=$ $84 \%, p<0.001$ ) (Fig. 4).

\section{Total WOMAC scores}

At 3 months, 6 studies reported total WOMAC scores and a statistically significant difference was found in favor of PRP treatment compared with control (MD, -14.53 [95\% CI, -21.97 to -7.09 ], $I^{2}=90 \%, p<0.001$ ). PRP treatment was also found to improve total WOMAC scores significantly according to the pooling analysis of 8 studies at 6 months (MD, -18.21 [95\% CI, -27.84 to -8.59 ], $\left.I^{2}=97 \%, p<0.001\right)$ and 4 studies at 12 months (MD, -19.45 [95\% CI, -26.09 to -12.82 ], $I^{2}=$ $85 \%, p<0.001$ ) (Fig. 5). 


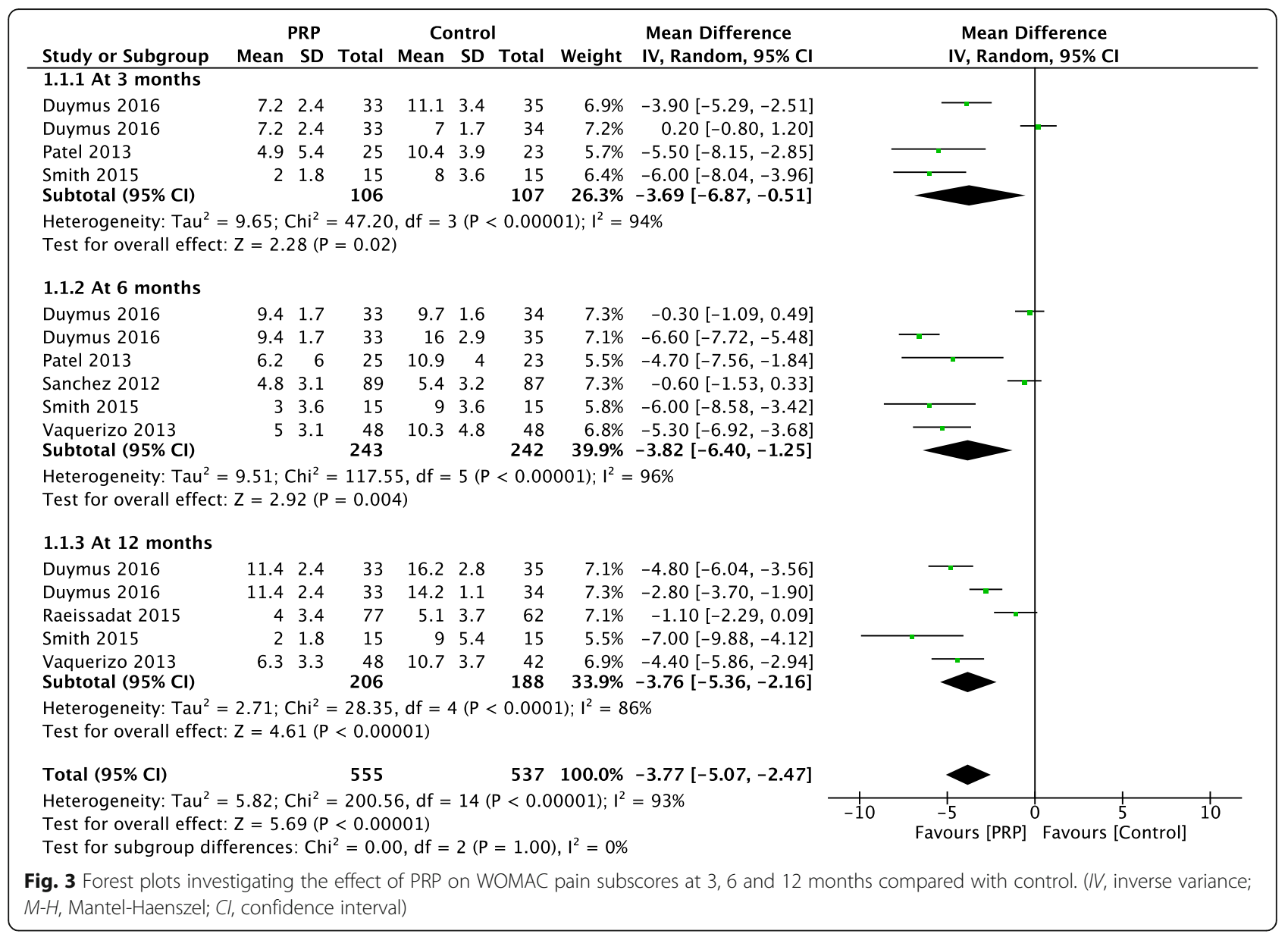

\section{Adverse events}

A total of 10 studies [9-11, 13-15, 17, 32, 34, 35] recorded adverse events. Excluding the study by Filardo et al. [17], which reported adverse events in a different form, there was no statistically significant difference in the number of patients with adverse events between PRP and HA among the rest 9 studies (RR, 1.40 [95\% CI, 0.80 to 2.45$], I^{2}=59 \%, p=0.24$ ) (Fig. 6). All adverse events were non-specific, the symptoms including pain, stiffness, syncope, dizziness, headache, nausea, gastritis, sweating, and tachycardia. No severe complications were recorded and all the events were self-resolved in days.

\section{Discussion}

This systematic review included 14 RCTs and assessed the temporal effect of PRP on knee pain and physical function in the treatment of knee OA compared with other intra-articular injections, including saline, HA, ozone, and corticosteroids. Data synthesis consistently showed intra-articular PRP injections significantly reduced knee pain, improved physical function, and total WOMAC scores compared with control. Such superiority was observed at 3, 6, and 12 months after treatment. However, the risk of adverse events in PRP-treated participants was not significantly increased in comparison with other intra-articular injections.

Although previous systematic reviews concluded that PRP was an effective and safe alternative to treat knee OA, such conclusion was reached on the basis of less than 9 RCTs [18-28], and thus the temporal effect of PRP injections on knee pain and physical function was not fully investigated. Chang et al. calculated the effect size of PRP treatment from different outcome measurements at 2, 6, and 12 follow-up, but half of the 16 studies included for analysis were case series, and 5 were RCTs [19]. Another systematic review pooled 6 RCTs and found that PRP obtained significantly better WOMAC total scores than HA from 3 to 12 months post-injection, however, only 2 studies reported WOMAC scores at 3 months and another 2 at 12 months [22]. Laudy et al. specifically evaluated the effect of PRP injections on knee pain and physical function at 6 and 12 months post-treatment [21]. Nonetheless, most comparisons included only 1 or 2 studies due to the small number of RCTs pooled for 


\begin{tabular}{|c|c|c|c|c|c|c|c|c|c|c|}
\hline \multirow{3}{*}{$\begin{array}{l}\text { Study or Subgroup } \\
1.2 .1 \text { At } 3 \text { months }\end{array}$} & \multicolumn{3}{|c|}{ PRP } & \multicolumn{3}{|c|}{ Control } & \multirow[b]{2}{*}{ Weight } & \multirow{3}{*}{$\begin{array}{l}\text { Mean Difference } \\
\text { IV, Random, } 95 \% \mathrm{Cl}\end{array}$} & \multirow{2}{*}{\multicolumn{2}{|c|}{$\begin{array}{c}\text { Mean Difference } \\
\text { IV, Random, } 95 \% \mathrm{CI}\end{array}$}} \\
\hline & Mean & SD & Total & Mean & SD & Total & & & & \\
\hline & & & & & & & & & & \\
\hline Duymus 2016 & 22 & 5.4 & 33 & 38.7 & 12.2 & 35 & $6.9 \%$ & $-16.70[-21.14,-12.26]$ & & \\
\hline Duymus 2016 & 22 & 5.4 & 33 & 25.1 & 8.9 & 34 & $7.1 \%$ & $-3.10[-6.61,0.41]$ & & \\
\hline Patel 2013 & 18.8 & 17.2 & 25 & 37.4 & 13.6 & 23 & $5.8 \%$ & $-18.60[-27.34,-9.86]$ & & \\
\hline $\begin{array}{l}\text { Smith } 2015 \\
\text { Subtotal }(95 \% \mathrm{Cl})\end{array}$ & 7 & 9 & $\begin{array}{r}15 \\
106\end{array}$ & 27 & 10.8 & $\begin{array}{r}15 \\
107\end{array}$ & $\begin{array}{r}6.3 \% \\
26.0 \%\end{array}$ & $\begin{array}{l}-20.00[-27.11,-12.89] \\
-\mathbf{1 4 . 2 4}[-23.43,-5.05]\end{array}$ & & \\
\hline \multicolumn{11}{|c|}{$\begin{array}{l}\text { Heterogeneity: } \mathrm{Tau}^{2}=78.05 ; \mathrm{Chi}^{2}=34.67, \mathrm{df}=3(\mathrm{P}<0.00001) ; \mathrm{I}^{2}=91 \% \\
\text { Test for overall effect: } Z=3.04(\mathrm{P}=0.002)\end{array}$} \\
\hline \multicolumn{11}{|l|}{ 1.2.2 At 6 months } \\
\hline Duymus 2016 & 29.6 & 5.7 & 33 & 54.1 & 7.3 & 35 & $7.1 \%$ & $-24.50[-27.60,-21.40]$ & - & \\
\hline Duymus 2016 & 29.6 & 5.7 & 33 & 30.1 & 5.7 & 34 & $7.2 \%$ & $-0.50[-3.23,2.23]$ & & \\
\hline Patel 2013 & 22.4 & 18.3 & 25 & 39.5 & 13 & 23 & $5.8 \%$ & $-17.10[-26.03,-8.17]$ & & \\
\hline Sanchez 2012 & 16.9 & 10.8 & 89 & 17.6 & 11.7 & 87 & $7.1 \%$ & $-0.70[-4.03,2.63]$ & & \\
\hline Smith 2015 & 8 & 10.8 & 15 & 31 & 10.8 & 15 & $6.1 \%$ & $-23.00[-30.73,-15.27]$ & & \\
\hline Vaquerizo 2013 & 19.7 & 11.1 & 48 & 36.2 & 16.8 & 48 & $\begin{array}{r}6.6 \% \\
39 \%\end{array}$ & $-16.50[-22.20,-10.80]$ & & \\
\hline \multicolumn{11}{|c|}{$\begin{array}{l}\text { Heterogeneity: } \text { Tau }^{2}=155.96 ; \mathrm{Chi}^{2}=178.57, \mathrm{df}=5(\mathrm{P}<0.00001) ; \mathrm{I}^{2}=97 \% \\
\text { Test for overall effect: } Z=2.58(P=0.010)\end{array}$} \\
\hline \multicolumn{11}{|l|}{ 1.2.3 At 12 months } \\
\hline Duymus 2016 & 38.6 & 7.7 & 33 & 54.2 & 7.9 & 35 & $7.0 \%$ & $-15.60[-19.31,-11.89]$ & & \\
\hline Duymus 2016 & 38.6 & 7.7 & 33 & 49.6 & 3.3 & 34 & $7.2 \%$ & $-11.00[-13.85,-8.15]$ & & \\
\hline Raeissadat 2015 & 13.2 & 10.4 & 77 & 19.5 & 11.9 & 62 & $7.0 \%$ & $-6.30[-10.06,-2.54]$ & & \\
\hline Smith 2015 & 7 & 7.2 & 15 & 30 & 12.6 & 15 & $6.2 \%$ & $-23.00[-30.34,-15.66]$ & & \\
\hline $\begin{array}{l}\text { Vaquerizo } 2013 \\
\text { Subtotal }(95 \% \mathrm{Cl})\end{array}$ & 21.9 & 11.3 & $\begin{array}{r}48 \\
206\end{array}$ & 38.9 & 14.2 & $\begin{array}{r}42 \\
188\end{array}$ & $\begin{array}{r}6.7 \% \\
34.1 \%\end{array}$ & $\begin{array}{l}-17.00[-22.35,-11.65] \\
\mathbf{- 1 3 . 9 6}[\mathbf{- 1 8 . 6 4}, \mathbf{- 9 . 2 8}]\end{array}$ & & \\
\hline \multicolumn{11}{|c|}{$\begin{array}{l}\text { Heterogeneity: } \text { Tau }^{2}=22.90 ; \mathrm{Chi}^{2}=24.71, \mathrm{df}=4(\mathrm{P}<0.0001) ; \mathrm{I}^{2}=84 \% \\
\text { Test for overall effect: } Z=5.85(P<0.00001)\end{array}$} \\
\hline Total $(95 \% \mathrm{Cl})$ & & & 555 & & & 537 & $100.0 \%$ & $-13.91[-18.53,-9.28]$ & & \\
\hline \multicolumn{9}{|c|}{$\begin{array}{l}\text { Heterogeneity: } \mathrm{Tau}^{2}=75.66 ; \mathrm{Chi}^{2}=242.65, \mathrm{df}=14(\mathrm{P}<0.00001) ; \mathrm{I}^{2} \\
\text { Test for overall effect: } \mathrm{Z}=5.90(\mathrm{P}<0.00001) \\
\text { Test for subgroup differences: } \mathrm{Chi}^{2}=0.01, \mathrm{df}=2(\mathrm{P}=0.99), \mathrm{I}^{2}=0 \%\end{array}$} & 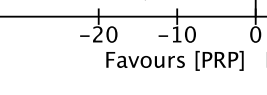 & 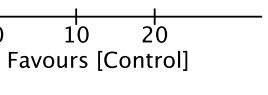 \\
\hline \multicolumn{11}{|c|}{$\begin{array}{l}\text { Fig. } 4 \text { Forest plots investigating the effect of PRP on WOMAC physical function subscores at 3, 6, and } 12 \text { months compared with control. (IV, inverse } \\
\text { variance; } M-H \text {, Mantel-Haenszel; Cl, confidence interval) }\end{array}$} \\
\hline
\end{tabular}

analysis. Another review included 9 RCTs and synthesized the WOMAC pain subscores and physical function subscores to compare the efficacy of PRP with control [23]. Due to the varied follow-up among studies, synthesis of the data at the latest follow-up might not reflect the changes of PRP efficacy. The strength of this study was to assess the effect of PRP treatment on knee pain and physical function at different time-points post-injection based on a larger number of RCTs.

It remains unclear regarding the duration period of the beneficial effect of PRP injections. Our study found that PRP was superior to other intra-articular injections in terms of pain relief and function improvement through 3 to 12 months. Filardo et al. investigated the persistence of the favorable effect of PRP infiltration during a 24-month follow-up [42]. Results show that all the evaluated parameters were significantly reduced at 24 months compared with those at 12 months, but still better than the baseline before treatment. The median duration of the clinical improvement was 9 months. This may explain why all current RCTs followed participants within 12 months. The short-term efficacy of PRP injections indicates that PRP only temporarily influences the joint milieu, without affecting the joint structure or progression of knee OA.

There are a few limitations in this review. The placebo effect was reportedly substantial in the treatment of knee $\mathrm{OA}$, especially in terms of pain relief and self-reported function improvement [43]. Interventions that are recently "hot" or that were administered through needles, such as intra-articular injections, would result in larger placebo effect [44]. Therefore, blinding of participants is critical to minimize the potential placebo effect. Half of the 14 RCTs in this review were believed to have successfully performed blinding of participants [13, 17, 29-35] according to the risk of bias assessment. While 2 more studies $[11,15]$ stated blinding of participants, the difference in injection times between the intervention and control groups actually made it difficult to perform blinding reliably. So future RCTs should be designed as double-blinding, which ought to be performed successfully during the whole trials. Another limitation is the high heterogeneity among studies, which was also common in previous reviews [18-28]. 


\begin{tabular}{|c|c|c|c|c|c|c|c|c|c|}
\hline \multirow{3}{*}{$\begin{array}{l}\text { Study or Subgroup } \\
1.3 .1 \text { At } 3 \text { months }\end{array}$} & \multirow{3}{*}{ Mean } & \multicolumn{2}{|l|}{ PRP } & \multicolumn{3}{|c|}{ Control } & \multirow[b]{2}{*}{ Weight } & \multirow{3}{*}{$\begin{array}{l}\text { Mean Difference } \\
\text { IV, Random, } 95 \% \mathrm{Cl}\end{array}$} & \multirow{2}{*}{$\begin{array}{c}\text { Mean Difference } \\
\text { IV, Random, } 95 \% \mathrm{Cl}\end{array}$} \\
\hline & & SD & Total & Mean & SD & Total & & & \\
\hline & & & & & & & & & \\
\hline Cerza 2012 & 39.1 & 17.8 & 60 & 57 & 11.7 & 60 & $4.9 \%$ & $-17.90[-23.29,-12.51]$ & \\
\hline Duymus 2016 & 32.2 & 7.8 & 33 & 53.1 & 15.9 & 35 & $4.9 \%$ & $-20.90[-26.80,-15.00]$ & \\
\hline Duymus 2016 & 32.2 & 7.8 & 33 & 35.3 & 10.5 & 34 & $5.0 \%$ & $-3.10[-7.52,1.32]$ & \\
\hline Li 2011 & 13.3 & 9.4 & 15 & 13.8 & 4.7 & 15 & $5.0 \%$ & $-0.50[-5.82,4.82]$ & \\
\hline Patel 2013 & 25.7 & 24.1 & 25 & 50.7 & 18.4 & 23 & $4.0 \%$ & $-25.00[-37.07,-12.93]$ & \\
\hline Smith 2015 & 10 & 12.6 & 15 & 37 & 12.6 & 15 & $4.5 \%$ & $-27.00[-36.02,-17.98]$ & \\
\hline $\begin{array}{l}\text { Spakova } 2012 \\
\text { Subtotal }(95 \% \mathrm{Cl})\end{array}$ & 14.4 & 14.2 & $\begin{array}{r}60 \\
241\end{array}$ & 26.2 & 17.5 & $\begin{array}{r}60 \\
242\end{array}$ & $\begin{array}{r}4.9 \% \\
33.2 \%\end{array}$ & $\begin{array}{r}-11.80[-17.50,-6.10] \\
-14.53[-21.97,-7.09]\end{array}$ & \\
\hline \multicolumn{10}{|c|}{$\begin{array}{l}\text { Heterogeneity: } \text { Tau }^{2}=88.05 ; \mathrm{Chi}^{2}=61.60, \mathrm{df}=6(\mathrm{P}<0.00001) ; \mathrm{I}^{2}=90 \% \\
\text { Test for overall effect: } Z=3.83(P=0.0001)\end{array}$} \\
\hline \multicolumn{10}{|l|}{ 1.3.2 At 6 months } \\
\hline Cerza 2012 & 36.5 & 17.9 & 60 & 65.1 & 10.6 & 60 & $5.0 \%$ & $-28.60[-33.86,-23.34]$ & $\longrightarrow$ \\
\hline Duymus 2016 & 42.8 & 7.1 & 33 & 76.6 & 10.7 & 35 & $5.1 \%$ & $-33.80[-38.09,-29.51]$ & - \\
\hline Duymus 2016 & 42.8 & 7.1 & 33 & 44.5 & 6.6 & 34 & $5.1 \%$ & $-1.70[-4.98,1.58]$ & 7 \\
\hline Li 2011 & 10.7 & 9.9 & 15 & 20.6 & 8.3 & 15 & $4.8 \%$ & $-9.90[-16.44,-3.36]$ & \\
\hline Patel 2013 & 30.5 & 25.9 & 25 & 53.1 & 17.9 & 23 & $3.9 \%$ & $-22.60[-35.11,-10.09]$ & \\
\hline Sanchez 2012 & 23.7 & 13.7 & 89 & 25.1 & 15.4 & 87 & $5.1 \%$ & $-1.40[-5.71,2.91]$ & \\
\hline Smith 2015 & 11 & 14.4 & 15 & 44 & 14.4 & 15 & $4.3 \%$ & $-33.00[-43.31,-22.69]$ & \\
\hline Spakova 2012 & 18.9 & 14.1 & 60 & 30.9 & 16.6 & 60 & $4.9 \%$ & $-12.00[-17.51,-6.49]$ & \\
\hline $\begin{array}{l}\text { Vaquerizo } 2013 \\
\text { Subtotal }(95 \% \mathrm{Cl})\end{array}$ & 27.2 & 15.1 & $\begin{array}{r}48 \\
378\end{array}$ & 50.4 & 23.2 & $\begin{array}{r}48 \\
377\end{array}$ & $\begin{array}{r}4.6 \% \\
42.8 \%\end{array}$ & $\begin{array}{l}-23.20[-31.03,-15.37] \\
-\mathbf{1 8 . 2 1}[-\mathbf{2 7 . 8 4 , - 8 . 5 9}]\end{array}$ & \\
\hline \multicolumn{10}{|c|}{$\begin{array}{l}\text { Heterogeneity: } \text { Tau }^{2}=204.01 ; \mathrm{Chi}^{2}=220.14, \mathrm{df}=8(P<0.00001) ; \mathrm{I}^{2}=96 \% \\
\text { Test for overall effect: } Z=3.71(\mathrm{P}=0.0002)\end{array}$} \\
\hline \multicolumn{10}{|l|}{ 1.3.3 At 12 months } \\
\hline Duymus 2016 & 54.9 & 10.8 & 33 & 77 & 10.1 & 35 & $5.0 \%$ & $-22.10[-27.08,-17.12]$ & \\
\hline Duymus 2016 & 54.9 & 10.8 & 33 & 69.3 & 4.3 & 34 & $5.1 \%$ & $-14.40[-18.36,-10.44]$ & \\
\hline Raeissadat 2015 & 18.4 & 14.4 & 77 & 27.5 & 16.4 & 62 & $5.0 \%$ & $-9.10[-14.30,-3.90]$ & \\
\hline Smith 2015 & 10 & 10.8 & 15 & 43 & 18.1 & 15 & $4.2 \%$ & $-33.00[-43.67,-22.33]$ & \\
\hline $\begin{array}{l}\text { Vaquerizo } 2013 \\
\text { Subtotal }(95 \% \mathrm{Cl})\end{array}$ & 30.8 & 15.5 & $\begin{array}{r}48 \\
206\end{array}$ & 54.2 & 19.2 & $\begin{array}{r}42 \\
188\end{array}$ & $\begin{array}{r}4.7 \% \\
24.0 \%\end{array}$ & $\begin{array}{r}-23.40[-30.68,-16.12] \\
\mathbf{- 1 9 . 4 5}[-\mathbf{2 6 . 0 9}, \mathbf{- 1 2 . 8 2}]\end{array}$ & \\
\hline \multicolumn{10}{|c|}{$\begin{array}{l}\text { Heterogeneity: } \text { Tau }^{2}=46.40 ; \mathrm{Chi}^{2}=26.18, \mathrm{df}=4(\mathrm{P}<0.0001) ; \mathrm{I}^{2}=85 \% \\
\text { Test for overall effect: } Z=5.75(P<0.00001)\end{array}$} \\
\hline Total $(95 \% \mathrm{Cl})$ & & & 825 & & & 807 & $100.0 \%$ & $-17.39[-22.32,-12.46]$ & \\
\hline \multicolumn{9}{|c|}{$\begin{array}{l}\text { Heterogeneity: } \mathrm{Tau}^{2}=120.45 ; \mathrm{Chi}^{2}=319.48, \mathrm{df}=20(\mathrm{P}<0.00001) ; \mathrm{I}^{2}=94 \% \\
\text { Test for overall effect: } Z=6.91(P<0.00001) \\
\text { Test for subgroup differences: } \mathrm{Chi}^{2}=0.97, \mathrm{df}=2(\mathrm{P}=0.62), \mathrm{I}^{2}=0 \%\end{array}$} & 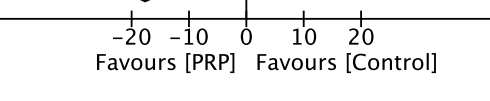 \\
\hline \multicolumn{10}{|c|}{$\begin{array}{l}\text { Fig. } 5 \text { Forest plots investigating the effect of PRP on total WOMAC scores at 3, 6, and } 12 \text { months compared with control. (IV, inverse variance; } \\
M-H \text {, Mantel-Haenszel; Cl, confidence interval) }\end{array}$} \\
\hline
\end{tabular}

\begin{tabular}{|c|c|c|c|c|c|c|c|c|c|c|}
\hline \multirow{2}{*}{$\frac{\text { Study or Subgroup }}{\text { Cerza } 2012}$} & $\begin{array}{l}\text { PRP } \\
\text { Events }\end{array}$ & Total & \multicolumn{2}{|c|}{ Control } & Weight & \multirow{2}{*}{$\begin{array}{c}\text { Risk Ratio } \\
\text { M-H, Random, } 95 \% \mathrm{Cl} \\
\text { Not estimable }\end{array}$} & \multicolumn{4}{|c|}{$\begin{array}{c}\quad \text { Risk Ratio } \\
\text { M-H, Random, } 95 \% \mathrm{Cl} \\
\end{array}$} \\
\hline & 0 & 60 & 0 & 60 & & & & & & \\
\hline Li 2011 & 12 & 15 & 12 & 15 & $29.4 \%$ & $1.00[0.70,1.43]$ & & & & \\
\hline Montanez-Heredia 2016 & 9 & 28 & 4 & 27 & $15.3 \%$ & $2.17[0.76,6.22]$ & & & & \\
\hline Patel 2013 & 11 & 25 & 0 & 23 & $3.6 \%$ & $21.23[1.32,341.04]$ & & & & \\
\hline Paterson 2016 & 2 & 12 & 0 & 11 & $3.3 \%$ & $4.62[0.25,86.72]$ & & & & \\
\hline Sanchez 2012 & 26 & 89 & 24 & 87 & $27.1 \%$ & $1.06[0.66,1.69]$ & & & - & \\
\hline Smith 2015 & 0 & 15 & 0 & 15 & & Not estimable & & & & \\
\hline Spakova 2012 & 6 & 60 & 0 & 60 & $3.4 \%$ & $13.00[0.75,225.75]$ & & & & \\
\hline Vaquerizo 2013 & 7 & 48 & 9 & 48 & $17.9 \%$ & $0.78[0.32,1.92]$ & & T & - & \\
\hline Total $(95 \% \mathrm{Cl})$ & & 352 & & 346 & $100.0 \%$ & $1.40[0.80,2.45]$ & & & & \\
\hline Total events & 73 & & 49 & & & & & & & \\
\hline $\begin{array}{l}\text { Heterogeneity: } \operatorname{Tau}^{2}=0 \\
\text { Test for overall effect: } Z\end{array}$ & $\begin{array}{l}; \mathrm{Chi}^{2}= \\
1.19(\mathrm{P}=\end{array}$ & $\begin{array}{l}14.58 \\
0.24)\end{array}$ & $\mathrm{df}=6(\mathrm{P}$ & $=0.02$ & 2); $I^{2}=59$ & & 0.005 & ${ }_{\text {Favours [PRP] }}^{0.1}$ & $\begin{array}{c}10 \\
\text { Favours [Control] }\end{array}$ & 200 \\
\hline
\end{tabular}




\section{Conclusions}

Intra-articular PRP injections probably are more efficacious in the treatment of knee OA in terms of pain relief and self-reported function improvement at 3, 6, and 12 months follow-up, compared with other injections, including saline placebo, HA, ozone, and corticosteroids.

\section{Additional files}

Additional file 1: Intervention protocol. (PDF $119 \mathrm{~kb}$ )
Additional file 2: PRISMA checklist. (DOC $64 \mathrm{~kb})$
Additional file 3: Search strategy and study selection. (PDF $84 \mathrm{~kb}$ )
Additional file 4: Characteristics and risk of bias assessment of included
and excluded studies. (PDF $462 \mathrm{~kb}$ )

\section{Abbreviations}

Cl: Confidence interval; CS: Corticosteroids; HA: Hyaluronic acid; IKDC: International knee documentation committee; KOOS: Knee injury and osteoarthritis outcome scores; LP-PRP: Leukocyte-poor PRP; LR-PRP: Leukocyte-rich PRP; MD: Mean difference; OA: Osteoarthritis; PRP: Platelet-rich plasma; RCTs: Randomized controlled trials; RR: Relative risk; WOMAC: Western Ontario and McMaster Universities Arthritis Index; VAS: Visual analogue scale

\section{Acknowledgements}

We thank Prof. Mandeep S. Dhillon, Prof. Gholam Reza Rassi, and Prof. Elham Ghorbani for their generous sharing of their unpublished data or information.

\section{Funding}

This study was funded by the National Natural Science Foundation of China (Grant No. 81401799) and Shanghai Youth Science and Technology Start-up Grants (Grant No. 14YF1412100).

\section{Availability of data and materials}

All data generated or analyzed during this study are either included in this published article or its supplementary information files.

\section{Authors' contributions}

LS, TY, SC, XX, and CZ conceived and designed the experiments. LS, TY, and $X X$ searched and screened the studies .LS, TY, and SC extracted and analyzed the data. $L S, T Y, S C, X X$, and $C Z$ wrote and revised the manuscript. All authors read and approved the final manuscript.

\section{Competing interests}

The authors declare that they have no competing interests.

\section{Consent for publication}

Not applicable.

\section{Ethics approval and consent to participate}

Not applicable.

\section{Author details}

'Department of Orthopaedic Surgery, Shanghai Sixth People's Hospital affiliated to Shanghai Jiaotong University School of Medicine, 600 Yishan Road, Shanghai 200233, China. ${ }^{2}$ Section of Clinical Epidemiology, Institute of Orthopaedic Traumatology affiliated to Shanghai Jiaotong University, 600 Yishan Road, Shanghai 200233, China.

Received: 18 November 2016 Accepted: 3 January 2017 Published online: 23 January 2017

\section{References}

1. Andia I, Maffulli N. Platelet-rich plasma for managing pain and inflammation in osteoarthritis. Nat Rev Rheumatol. 2013;9:721-30.

2. Jevsevar DS, Brown GA, Jones DL, Matzkin EG, Manner PA, Mooar P, et al. The American Academy of Orthopaedic Surgeons evidence-based guideline on: treatment of osteoarthritis of the knee, 2nd edition. J Bone Joint Surg Am. 2013;95:1885-6.

3. McAlindon TE, Bannuru RR, Sullivan MC, Arden NK, Berenbaum F, BiermaZeinstra SM, et al. OARSI guidelines for the non-surgical management of knee osteoarthritis. Osteoarthritis Cartilage. 2014;22:363-88.

4. Hashemi M, Jalili P, Mennati S, Koosha A, Rohanifar R, Madadi F, et al. The effects of prolotherapy with hypertonic dextrose versus prolozone (intraarticular Ozone) in patients with knee osteoarthritis. Anesth Pain Med. 2015;5, e27585.

5. Cugat R, Cusco X, Seijas R, Alvarez P, Steinbacher G, Ares O, et al. Biologic enhancement of cartilage repair: the role of platelet-rich plasma and other commercially available growth factors. Arthroscopy. 2015;31:777-83.

6. Hsu WK, Mishra A, Rodeo SR, Fu F, Terry MA, Randelli P, et al. Platelet-rich plasma in orthopaedic applications: evidence-based recommendations for treatment. J Am Acad Orthop Surg. 2013;21:739-48.

7. Smyth NA, Murawski CD, Fortier LA, Cole BJ, Kennedy JG. Platelet-rich plasma in the pathologic processes of cartilage: review of basic science evidence. Arthroscopy. 2013;29:1399-409.

8. Xie X, Zhang C, Tuan RS. Biology of platelet-rich plasma and its clinical application in cartilage repair. Arthritis Res Ther. 2014;16:204.

9. Cerza F, Carni S, Carcangiu A, Di Vavo I, Schiavilla V, Pecora A, et al. Comparison between hyaluronic acid and platelet-rich plasma, intra-articular infiltration in the treatment of gonarthrosis. Am J Sports Med. 2012;40:2822-7.

10. Li M, Zhang C, Ai Z, Yuan T, Feng Y, Jia W. Therapeutic effectiveness of intra-knee-articular injection of platelet-rich plasma on knee articular cartilage degeneration. Zhongguo Xiu Fu Chong Jian Wai Ke Za Zhi. 2011;25:1192-6.

11. Patel S, Dhillon MS, Aggarwal S, Marwaha N, Jain A. Treatment with platelet-rich plasma is more effective than placebo for knee osteoarthritis: a prospective, double-blind, randomized trial. Am J Sports Med. 2013;41:356-64.

12. Rayegani SM, Raeissadat SA, Taheri MS, Babaee M, Bahrami MH, Eliaspour D, et al. Does intra articular platelet rich plasma injection improve function, pain and quality of life in patients with osteoarthritis of the knee? A randomized clinical trial. Orthop Rev (Pavia). 2014;6:5405.

13. Sanchez M, Fiz N, Azofra J, Usabiaga J, Aduriz Recalde E, Garcia Gutierrez A, et al. A randomized clinical trial evaluating plasma rich in growth factors (PRGF-Endoret) versus hyaluronic acid in the short-term treatment of symptomatic knee osteoarthritis. Arthroscopy. 2012;28:1070-8.

14. Spakova T, Rosocha J, Lacko M, Harvanova D, Gharaibeh A. Treatment of knee joint osteoarthritis with autologous platelet-rich plasma in comparison with hyaluronic acid. Am J Phys Med Rehabil. 2012;91:411-7.

15. Vaquerizo V, Plasencia MA, Arribas I, Seijas R, Padilla S, Orive G, et al. Comparison of intra-articular injections of plasma rich in growth factors (PRGF-Endoret) versus Durolane hyaluronic acid in the treatment of patients with symptomatic osteoarthritis: a randomized controlled trial. Arthroscopy. 2013;29:1635-43.

16. Filardo G, Kon E, Di Martino A, Di Matteo B, Merli ML, Cenacchi A, et al. Platelet-rich plasma vs hyaluronic acid to treat knee degenerative pathology: study design and preliminary results of a randomized controlled trial. BMC Musculoskelet Disord. 2012;13:229

17. Filardo G, Di Matteo B, Di Martino A, Merli ML, Cenacchi A, Fornasari P, et al. Platelet-rich plasma intra-articular knee injections show no superiority versus viscosupplementation: a randomized controlled trial. Am J Sports Med. 2015;43:1575-82.

18. Anitua E, Sanchez M, Aguirre JJ, Prado R, Padilla S, Orive G. Efficacy and safety of plasma rich in growth factors intra-articular infiltrations in the treatment of knee osteoarthritis. Arthroscopy. 2014;30:1006-17.

19. Chang KV, Hung CY, Aliwarga F, Wang TG, Han DS, Chen WS. Comparative effectiveness of platelet-rich plasma injections for treating knee joint cartilage degenerative pathology: a systematic review and meta-analysis. Arch Phys Med Rehabil. 2014;95:562-75.

20. Khoshbin A, Leroux T, Wasserstein D, Marks P, Theodoropoulos J, OgilvieHarris D, et al. The efficacy of platelet-rich plasma in the treatment of symptomatic knee osteoarthritis: a systematic review with quantitative synthesis. Arthroscopy. 2013;29:2037-48.

21. Laudy AB, Bakker EW, Rekers M, Moen MH. Efficacy of platelet-rich plasma injections in osteoarthritis of the knee: a systematic review and metaanalysis. Br J Sports Med. 2015:49:657-72.

22. Meheux CJ, McCulloch PC, Lintner DM, Varner KE, Harris JD. Efficacy of intraarticular platelet-rich plasma injections in knee osteoarthritis: a systematic review. Arthroscopy. 2016;32:495-505. 
23. Kanchanatawan W, Arirachakaran A, Chaijenkij K, Prasathaporn N, Boonard M, Piyapittayanun $\mathrm{P}$, et al. Short-term outcomes of platelet-rich plasma injection for treatment of osteoarthritis of the knee. Knee Surg Sports Traumatol Arthrosc. 2016;24:1665-77.

24. Ornetti $P$, Nourissat $G$, Berenbaum F, Sellam J, Richette $P$, Chevalier $X$, under the aegis of the Osteoarthritis Section of the French Society for Rheumatology (Société Française de Rhumatologie, SFR). Does platelet-rich plasma have a role in the treatment of osteoarthritis? Joint Bone Spine. 2016:83:31-6.

25. Campbell KA, Saltzman BM, Mascarenhas R, Khair MM, Verma NN, Bach Jr $B R$, et al. Does intra-articular platelet-rich plasma injection provide clinically superior outcomes compared with other therapies in the treatment of knee osteoarthritis? A systematic review of overlapping meta-analyses. Arthroscopy. 2015;31:2213-21.

26. Lai LP, Stitik TP, Foye PM, Georgy JS, Patibanda V, Chen B. Use of plateletrich plasma in intra-articular knee injections for osteoarthritis: a systematic review. PM R. 2015;7:637-48.

27. LaPrade CM, James EW, LaPrade RF, Engebretsen L. How should we evaluate outcomes for use of biologics in the knee? J Knee Surg. 2015;28:35-44

28. Dold AP, Zywiel MG, Taylor DW, Dwyer T, Theodoropoulos J. Platelet-rich plasma in the management of articular cartilage pathology: a systematic review. Clin J Sport Med. 2014;24:31-43.

29. Duymus TM, Mutlu S, Dernek B, Komur B, Aydogmus S, Kesiktas FN. Choice of intra-articular injection in treatment of knee osteoarthritis: platelet-rich plasma, hyaluronic acid or ozone options. Knee Surg Sports Traumatol Arthrosc. 2016;doi: 10.1007/s00167-016-4110-5

30. Forogh B, Mianehsaz E, Shoaee S, Ahadi T, Raissi GR, Sajadi S. Effect of single injection of platelet-rich plasma in comparison with corticosteroid on knee osteoarthritis: a double-blind randomized clinical trial. J Sports Med Phys Fitness. 2016:56:901-8.

31. Gormeli G, Gormeli CA, Ataoglu B, Colak C, Aslanturk O, Ertem K. Multiple PRP injections are more effective than single injections and hyaluronic acid in knees with early osteoarthritis: a randomized, double-blind, placebocontrolled trial. Knee Surg Sports Traumatol Arthrosc. 2015; doi:10.1007/s00167-015-3705-6

32. Paterson KL, Nicholls M, Bennell KL, Bates D. Intra-articular injection of photo-activated platelet-rich plasma in patients with knee osteoarthritis: a double-blind, randomized controlled pilot study. BMC Musculoskelet Disord. 2016;17:67.

33. Raeissadat SA, Rayegani SM, Hassanabadi H, Fathi M, Ghorbani E, Babaee M, et al. Knee osteoarthritis injection choices: platelet- rich plasma (PRP) versus hyaluronic acid (a one-year randomized clinical trial). Clin Med Insights Arthritis Musculoskelet Disord. 2015;8:1-8.

34. Smith PA. Intra-articular autologous conditioned plasma injections provide safe and efficacious treatment for knee osteoarthritis: an FDA-sanctioned, randomized, double-blind, placebo-controlled clinical trial. Am J Sports Med. 2016:44:884-91.

35. Montanez-Heredia E, Irizar S, Huertas PJ, Otero E, Del Valle M, Prat I, et al. Intra-articular injections of platelet-rich plasma versus hyaluronic acid in the treatment of osteoarthritic knee pain: a randomized clinical trial in the context of the Spanish National Health Care System. Int J Mol Sci. 2016;17(7). doi: 10.3390/ijms17071064.

36. McConnell S, Kolopack P, Davis AM. The Western Ontario and McMaster Universities Osteoarthritis Index (WOMAC): a review of its utility and measurement properties. Arthritis Rheum. 2001;45:453-61.

37. Gobbi A, Lad D, Karnatzikos G. The effects of repeated intra-articular PRP injections on clinical outcomes of early osteoarthritis of the knee. Knee Surg Sports Traumatol Arthrosc. 2015;23:2170-7.

38. Kavadar G, Demircioglu DT, Celik MY, Emre TY. Effectiveness of platelet-rich plasma in the treatment of moderate knee osteoarthritis: a randomized prospective study. J Phys Ther Sci. 2015;27:3863-7.

39. Higgins JPT, Green S. Cochrane Handbook for Systematic Reviews of Interventions Version 5.1.0 [updated March 2011]: The Cochrane Collaboration; 2011. Available from: http://handbook.cochrane.org/.

40. Kellgren JH, Lawrence JS. Radiological assessment of osteo-arthrosis. Ann Rheum Dis. 1957;16:494-502.

41. Ahlback S. Osteoarthrosis of the knee. A radiographic investigation. Acta Radiol Diagn (Stockh). 1968:Suppl 277:7-72.
42. Filardo G, Kon E, Buda R, Timoncini A, Di Martino A, Cenacchi A, et al. Platelet-rich plasma intra-articular knee injections for the treatment of degenerative cartilage lesions and osteoarthritis. Knee Surg Sports Traumatol Arthrosc. 2011;19:528-35.

43. Zhang W, Robertson J, Jones AC, Dieppe PA, Doherty M. The placebo effect and its determinants in osteoarthritis: meta-analysis of randomised controlled trials. Ann Rheum Dis. 2008;67:1716-23.

44. Doherty M, Dieppe P. The "placebo" response in osteoarthritis and its implications for clinical practice. Osteoarthritis Cartilage. 2009;17:1255-62.

45. Riboh JC, Saltzman BM, Yanke AB, Fortier L, Cole BJ. Effect of leukocyte concentration on the efficacy of platelet-rich plasma in the treatment of knee osteoarthritis. Am J Sports Med. 2016;44:792-800.

\section{Submit your next manuscript to BioMed Central and we will help you at every step:}

- We accept pre-submission inquiries

- Our selector tool helps you to find the most relevant journal

- We provide round the clock customer support

- Convenient online submission

- Thorough peer review

- Inclusion in PubMed and all major indexing services

- Maximum visibility for your research

Submit your manuscript at www.biomedcentral.com/submit 